\title{
Tulane
}

\author{
Tulane Economics Working Paper Series
}

\section{Identification-Robust Minimum Distance Estimation of the New Keynesian Phillips Curve}

\author{
Leandro M. Magnusson \\ Department of Economics \\ 206 Tilton Hall \\ Tulane University \\ New Orleans, LA 70118 \\ Imagnuss@tulane.edu
}

\author{
Sophocles Mavroeidis \\ Department of Economics \\ Brown University \\ 64 Waterman Street \\ Providence, RI 02912 \\ Sophocles_Mavroeidis@brown.edu
}

Working Paper 0904

February 2009

\begin{abstract}
Limited-information identification-robust methods on the indexation and price rigidity parameters of the new Keynesian Phillips curve yield very wide confidence intervals. Full-information methods impose more restrictions on the reduced-form dynamics, and thus make more efficient use of the information in the data. We propose identification-robust minimum distance methods for exploiting these additional restrictions and show that they yield considerably smaller confidence intervals for the coefficients of the model compared to their limited-information GMM counterparts. In contrast to previous studies that used GMM, we find evidence of partial but not full indexation, and we obtain sharper inference on the degree of price stickiness.
\end{abstract}

Keywords: weak identification, minimum distance, GMM, Phillips curve JEL codes: C22, E31 


\title{
Identification-robust Minimum Distance estimation of the New Keynesian Phillips Curve
}

\author{
Leandro M. Magnusson \\ Tulane University \\ lmagnuss@tulane.edu
}

\author{
Sophocles Mavroeidis* \\ Brown University \\ Sophocles_Mavroeidis@Brown.edu
}

February 15, 2009

\begin{abstract}
Limited-information identification-robust methods on the indexation and price rigidity parameters of the new Keynesian Phillips curve yield very wide confidence intervals. Full-information methods impose more restrictions on the reduced-form dynamics, and thus make more efficient use of the information in the data. We propose identificationrobust minimum distance methods for exploiting these additional restrictions and show that they yield considerably smaller confidence intervals for the coefficients of the model compared to their limited-information GMM counterparts. In contrast to previous studies that used GMM, we find evidence of partial but not full indexation, and we obtain sharper inference on the degree of price stickiness.
\end{abstract}

Keywords: Weak identification, Minimum Distance, GMM, Phillips Curve JEL classification: C22, E31

\footnotetext{
${ }^{*}$ Corresponding author.
} 


\section{Introduction}

The new Keynesian Phillips curve (NKPC) is a forward-looking model of sticky prices, according to which inflation is driven by the expected discount stream of marginal costs. In its purely forward-looking specification, the model is at odds with US data over the postwar period, and it is typically replaced by a hybrid version, that includes also lagged inflation:

$$
\pi_{t}=\lambda x_{t}+\gamma_{f} E_{t}\left(\pi_{t+1}\right)+\gamma_{b} \pi_{t-1}+\varepsilon_{t},
$$

where $\pi_{t}$ denotes inflation, $x_{t}$ is a measure of marginal costs, $\varepsilon_{t}$ is an exogenous cost-push or markup shock (which could be zero), and $E_{t}$ denotes expectations conditional on information available at time $t$, see Woodford (2003) for details.

Equation (1) has been estimated in the literature using so-called limited- or full-information methods. Limited-information or 'single-equation' methods do not require the specification of a model for the forcing variable $x_{t}$, and their results are therefore more general than those derived using full-information methods. A prominent limited-information approach for the estimation of the NKPC, popularized in a seminal paper by Galí and Gertler (1999), is based on replacing expectations of future inflation in equation (1) by their realization. The resulting equation is estimated by the generalized method of moments (GMM), using predetermined variables as instruments. It is by now well-established that estimation of the NKPC may be subject to weak instrument problems, see for example Kleibergen and Mavroeidis (2009) and the references therein. Recently, a number of studies applied identification-robust GMM-based tests to the NKPC, and reported very wide confidence intervals, see Dufour et. al. (2006, 2008) and Kleibergen and Mavroeidis (2009).

In contrast, full-information methods specify a complete model for the dynamics of inflation and the forcing variable. Various full-information approaches have been used for the estimation of the NKPC, which differ in terms of whether the model of the forcing variable is structural or reduced-form, as well as in terms of econometric methodology, i.e., Minimum 
Distance (MD) or likelihood-based inference. One such approach used by Sbordone (2002, 2005) is MD estimation based on a reduced-form vector autoregressive model (VAR) for $\pi_{t}$ and $x_{t}{ }^{1}$ It is clear that this approach imposes more restrictions on the underlying data generating process than limited-information GMM, so we expect it to make more efficient use of the information in the data. Thus, in view of the aforementioned negative results on the identification of the NKPC, reviewed in Kleibergen and Mavroeidis (2009), our main objective in this paper is to investigate the extent to which imposing more restrictions on the reduced form dynamics sharpens inference on the NKPC parameters. We do so using the identification-robust methods for MD that were recently developed in a different setting by Magnusson (2008). These are the MD versions of the identification-robust GMM tests proposed by Stock and Wright (2000) and Kleibergen (2005). Even though the focus of the present study is on the Phillips curve, our paper also provides a methodological contribution towards the development of identification-robust methods for full-information inference in dynamic stochastic general equilibrium (DSGE) models. ${ }^{2}$

Our analysis starts with a discussion of the identification-robust MD methods, and a comparison with their limited-information GMM counterparts in the context of the NKPC. We show that the difference in the two approaches essentially lies in the way they use past data to proxy the expectations of future inflation. The difference is akin to the issue of iterative versus direct multistep forecasting, which has been studied by the forecasting literature, see e.g., Schorfheide (2005) and Marcellino, Stock, and Watson (2006). Iterative forecasting corresponds to what the MD approach does, and it is more efficient, albeit less robust, than the direct multistep forecasting, which corresponds to GMM. We report power curves that show considerable power advantages of MD over GMM. These results are consistent with our subsequent empirical findings.

\footnotetext{
${ }^{1} \mathrm{MD}$ was also used by Christiano, Eichenbaum, and Evans (2005) based on a structural model of the dynamics.

${ }^{2} \mathrm{~A}$ related study in this direction is the paper by Dufour et. al. (2008). This study differs from ours in that it does not impose assumptions on the reduced-form dynamics directly, but rather it exploits the correlation of the structural errors in a system of structural equations.
} 
Our empirical analysis is based on a microfounded version of the NKPC that was studied by Sbordone (2005), according to which the so-called 'semi-structural' parameters $\lambda, \gamma_{f}$ and $\gamma_{b}$ in equation (1) are functions of some deeper structural parameters that measure the degree of price stickiness and the degree of indexation of prices to past inflation. Following Sbordone (2005), we model the reduced-form dynamics of $\left(\pi_{t}, x_{t}\right)$ by a VAR, and we use the labor share as a proxy for marginal costs, in accordance with Galí and Gertler (1999). We estimate the model using quarterly data from the US economy over the period 1984 to 2008.

Our main finding is that exploiting the restrictions on the reduced-form dynamics improves the identification of the structural parameters of the NKPC considerably: the MD identification-robust confidence sets on the price rigidity and indexation parameters are about half the size of their GMM counterparts. Specifically, we find evidence of partial but not full indexation, so we can reject the pure forward-looking version of the NKPC, as well as the model with full indexation, which was used in Christiano, Eichenbaum, and Evans (2005). In terms of the price rigidity parameter, our confidence intervals are smaller than their GMM counterparts, but they are still fairly wide. This adds further weight to the view that the average duration of prices is hard to estimate accurately from aggregate macroeconomic data.

The structure of the paper is as follows. Section 2 presents the version of the NKPC model that we estimate and derives the identification-robust MD test statistics. Section 3 discusses the difference of the MD tests from their GMM counterparts. Section 4 reports the empirical results, and section 5 offers some concluding remarks. Proofs are given in the Appendix at the end.

\section{Identification-robust MD tests for the NKPC}

We shall consider here the structural parameterization of the NKPC derived from Calvo (1983) pricing and indexation, as in Sbordone (2002, 2005) and Christiano, Eichenbaum, 
and Evans (2005). According to this specification, the parameters $\lambda, \gamma_{f}$ and $\gamma_{b}$ are functions of the degree of price stickiness $\theta$, which is the probability that a firm will be unable to change its price in a given period, the indexation parameter $\varrho$, which measures the degree of indexation to past inflation, and the discount factor $\beta$. As explained in Woodford (2003), in the special case $\beta=1$, the coefficients $\gamma_{f}$ and $\gamma_{b}$ in equation (1) add to one. In this case, the model is identical to that of Fuhrer and Moore (1995), and also has identical implications to another popular version of the NKPC due to Galí and Gertler (1999), according to which there is no indexation, but a fraction of agents use a backward-looking rule of thumb to set their prices. Thus, in our empirical analysis we set $\beta$ equal to one. ${ }^{3}$ The model to be estimated is given by the equation

$$
\pi_{t}=\frac{(1-\theta)^{2}}{\theta(1+\varrho)} x_{t}+\frac{1}{1+\varrho} E_{t}\left(\pi_{t+1}\right)+\frac{\varrho}{1+\varrho} \pi_{t-1}+\varepsilon_{t}
$$

To evaluate the central predictions of this model, we follow the approach of Sbordone (2005) which is based on the distance between the dynamics of inflation implied by the structural model and the dynamics implied by a reduced-form VAR forecasting model. The latter can be written in companion form as:

$$
\mathbf{z}_{t}=A(\varphi) \mathbf{z}_{t-1}+\epsilon_{t}
$$

Let $e_{\pi}, e_{x}$ denote the unit vectors such that $e_{\pi}^{\prime} \mathbf{z}_{t}=\pi_{t}$ and $e_{x}^{\prime} \mathbf{z}_{t}=x_{t}$. Letting $k$ denote the number of variables in the VAR and $p$ the number of lags (order of the VAR), the reduced form coefficient matrix $A(\varphi)$ is of dimension $k p \times k p$, and it contains $k^{2} p$ unknown (reduced-form) parameters, denoted by $\varphi$.

Next, we need to link the reduced-form parameters $\varphi$ to the structural parameters $\vartheta=$ $(\theta, \varrho)^{\prime}$ in the NKPC (2). We use the standard identifying assumption in the literature

\footnotetext{
${ }^{3}$ We note that the results are insensitive to alternative values for $\beta$ near one. Moreover, Kleibergen and Mavroeidis (2009) report evidence that $\gamma_{f}+\gamma_{b}$ is very well identified and not significantly different from one.
} 
that $E_{t-1} \varepsilon_{t}=0$, see for example, Galí and Gertler (1999) and Sbordone (2002). ${ }^{4}$ Taking expectations with respect to information at $t-1$ on both sides of equation (1) yields

$$
E_{t-1} \pi_{t}=\frac{(1-\theta)^{2}}{\theta(1+\varrho)} E_{t-1}\left(x_{t}\right)+\frac{1}{1+\varrho} E_{t-1} \pi_{t+1}+\frac{\varrho}{1+\varrho} \pi_{t-1}
$$

Substituting for $E_{t-1} \pi_{t+1}=e_{\pi}^{\prime} A(\varphi)^{2} \mathbf{z}_{t-1}, E_{t-1} \pi_{t}=e_{\pi}^{\prime} A(\varphi) \mathbf{z}_{t-1}, E_{t-1} x_{t}=e_{x}^{\prime} A(\varphi) \mathbf{z}_{t-1}$ and $\pi_{t-1}=e_{\pi}^{\prime} \mathbf{z}_{t-1}$ yields

$$
\left\{e_{\pi}^{\prime}\left[I-\frac{1}{1+\varrho} A(\varphi)\right]-\frac{(1-\theta)^{2}}{\theta(1+\varrho)} e_{x}^{\prime}\right\} A(\varphi)-\frac{\varrho}{1+\varrho} e_{\pi}^{\prime}=0
$$

where $I$ denotes the identity matrix of dimension $k p$. The $k p$ restrictions (4) will be used to do inference on the structural parameters $\vartheta$ based on some estimator of the reduced-form parameters, $\hat{\varphi}$.

The restrictions (4) can be expressed using the distance function

$$
g(\varphi, \vartheta)=A(\varphi)^{\prime}\left\{\left[I-\frac{1}{1+\varrho} A(\varphi)^{\prime}\right] e_{\pi}-\frac{(1-\theta)^{2}}{\theta(1+\varrho)} e_{x}\right\}-\frac{\varrho}{1+\varrho} e_{\pi}
$$

We shall also make use of the following Jacobian matrices:

$$
G_{\varphi}(\varphi, \vartheta) \equiv \frac{\partial g(\varphi, \vartheta)}{\partial \varphi^{\prime}}, \quad \text { and } \quad G_{\vartheta}(\varphi, \vartheta) \equiv \frac{\partial g(\varphi, \vartheta)}{\partial \vartheta^{\prime}}
$$

Let $\hat{\varphi}$ denote a consistent and asymptotically normal estimator of the reduced-form parameters, with asymptotic variance matrix $V_{\varphi}$, and let $\hat{V}_{\varphi}$ be a consistent estimator of $V_{\varphi}$. By the Delta method, the asymptotic variance of $g(\hat{\varphi}, \vartheta)$ is $G_{\varphi}(\varphi, \vartheta) V_{\varphi} G_{\varphi}(\varphi, \vartheta)^{\prime}$. Efficient MD estimation is based on the criterion function

$$
Q(\vartheta)=g(\hat{\varphi}, \vartheta)^{\prime} \hat{V}_{g g}(\bar{\vartheta})^{-1} g(\hat{\varphi}, \vartheta)
$$

${ }^{4}$ This can be relaxed to allow for serial correlation in the cost push shock, e.g., $E_{t-1} \varepsilon_{t}=\rho_{\varepsilon} \varepsilon_{t-1}$, at the cost of introducing additional unknown parameters. 
where $\hat{V}_{g g}(\vartheta)=G_{\varphi}(\hat{\varphi}, \vartheta) \hat{V}_{\varphi} G_{\varphi}(\hat{\varphi}, \vartheta)^{\prime}$. The value of the parameter $\bar{\vartheta}$ that appears in the weight matrix $\hat{V}_{g g}(\bar{\vartheta})^{-1}$ may be some preliminary (inefficient) estimator of $\vartheta$, e.g., a MD estimator using the identity weight matrix, in which case the procedure corresponds to classical 2-step MD estimation, see Newey and McFadden (1994). However, $\bar{\vartheta}$ can also be equal to $\vartheta$, in which case the criterion function (7) corresponds to the so-called continuously updated (CU) GMM criterion function of Hansen, Heaton, and Yaron (1996).

Standard inference is based on the assumption that the Jacobian matrix $G_{\vartheta}(\varphi, \vartheta)$, see equation (6), is of full rank. Under this assumption, 2-step and CU-MD estimators are asymptotically equivalent, and since the former is computationally simpler, it is the one commonly used. In that case, the minimizer of the MD criterion function, $\hat{\vartheta}$ is asymptotically normal, and the usual Wald statistics for hypotheses on $\vartheta$ are asymptotically $\chi^{2}$ distributed, see Newey and McFadden (1994). However, when $G_{\vartheta}(\varphi, \vartheta)$ is nearly of reduced rank, these conventional asymptotic approximations break down, and inference based on Wald tests can be seriously misleading, see Stock et. al. (2002). Therefore, we consider here test statistics whose distribution under the null hypothesis $H_{0}: \vartheta=\vartheta_{0}$ is independent of the rank of the Jacobian matrix $G_{\vartheta}(\varphi, \vartheta)$, and as a result, inference based on these statistics is robust to weak identification or weak instrument problems. The difference of these statistics to their GMM counterparts will be highlighted in the next section.

The first test statistic we consider is the MD version of the statistic of Anderson and Rubin (1949) which is given by the CU-MD objective function, equation (7) with $\bar{\vartheta}=\vartheta$, scaled by the sample size:

$$
\operatorname{MD}-\operatorname{AR}\left(\vartheta_{0}\right)=T g\left(\hat{\varphi}, \vartheta_{0}\right)^{\prime} \hat{V}_{g g}\left(\vartheta_{0}\right)^{-1} g\left(\hat{\varphi}, \vartheta_{0}\right)
$$

Like the original Anderson-Rubin statistic, $A R_{M D}$ can be interpreted as a test of the validity of the restrictions implied by the model, see equation (4), at a given hypothesized value of the parameters $\left(\theta_{0}, \varrho_{0}\right)$. 
The second statistic is a score statistic that is based on the derivative of the CU-MD objective function. This statistic is the MD version of the $\mathrm{K}$ statistic proposed by Kleibergen (2002, 2005). It can be shown that

$$
\frac{1}{2} \frac{\partial Q(\vartheta)}{\partial \vartheta^{\prime}}=g(\hat{\varphi}, \vartheta)^{\prime} \hat{V}_{g g}(\vartheta)^{-1} \hat{D}(\vartheta)
$$

where

$$
\begin{aligned}
\hat{D}(\vartheta)= & {\left[\frac{\partial g(\hat{\varphi}, \theta, \varrho)}{\partial \theta}-\hat{V}_{\theta g}(\vartheta) \hat{V}_{g g}(\vartheta)^{-1} g(\hat{\varphi}, \vartheta),\right.} \\
& \left.\frac{\partial g(\hat{\varphi}, \theta, \varrho)}{\partial \varrho}-\hat{V}_{\varrho g}(\vartheta) \hat{V}_{g g}(\vartheta)^{-1} g(\hat{\varphi}, \vartheta)\right] .
\end{aligned}
$$

$\hat{V}_{\theta g}(\vartheta)=\frac{\partial G_{\varphi}(\hat{\varphi}, \vartheta)}{\partial \theta} \hat{V}_{\varphi} G_{\varphi}(\hat{\varphi}, \vartheta)^{\prime}$ and $\hat{V}_{\varrho g}(\vartheta)=\frac{\partial G_{\varphi}(\hat{\varphi}, \vartheta)}{\partial \varrho} \hat{V}_{\varphi} G_{\varphi}(\hat{\varphi}, \vartheta)^{\prime}$. The matrix $\hat{D}(\vartheta)$ can be thought of as an alternative estimator of the Jacobian $G_{\vartheta}(\varphi, \vartheta)$, which is asymptotically independent of $g(\hat{\varphi}, \vartheta)$. The K statistic for testing the hypothesis $H_{0}: \vartheta=\vartheta_{0}$ is given by a quadratic form of the derivative of the CU-MD objective function (9) with respect to an estimator of its variance, i.e.:

$$
\operatorname{MD}-\mathrm{K}\left(\vartheta_{0}\right)=g\left(\hat{\varphi}, \vartheta_{0}\right)^{\prime} \hat{V}_{g g}\left(\vartheta_{0}\right)^{-\frac{1}{2}} P_{\hat{V}_{g g}\left(\vartheta_{0}\right)^{-\frac{1}{2}} \hat{D}\left(\vartheta_{0}\right)} \hat{V}_{g g}\left(\vartheta_{0}\right)^{-\frac{1}{2}} g\left(\hat{\varphi}, \vartheta_{0}\right)
$$

where $P_{X}=X\left(X^{\prime} X\right)^{-1} X^{\prime}$ for any full rank matrix $X$.

Note that, like in the case of GMM, because the MD-K statistic depends on the derivative of the objective function, tests based on it may suffer a spurious decline of power at inflection points of the objective function, where the identifying restrictions are typically violated. Thus, we shall follow the approach of Kleibergen (2005) and combine the MD-K statistic with a statistic that tests the validity of the overidentifying restrictions under $H_{0}$ :

$$
\operatorname{MD}-J\left(\vartheta_{0}\right)=\operatorname{MD}-\operatorname{AR}\left(\vartheta_{0}\right)-\operatorname{MD}-K\left(\vartheta_{0}\right) \text {. }
$$

The joint test will be referred to as MD-KJ test, and it will reject the null hypothesis at the $\alpha \%$ significance level whenever the hypothesis is rejected either by an $\alpha_{1} \%$ level MD-K test 
or an $\alpha_{2} \%$ MD-J test, where $\alpha_{1}+\alpha_{2}=\alpha$. In our empirical analysis below, we use $\alpha_{1}=.8 \alpha$ and $\alpha_{2}=.2 \alpha$.

The following result gives the asymptotic distribution of these statistics under the null hypothesis $H_{0}: \vartheta=\vartheta_{0}$.

Proposition 1 Assume that $\sqrt{T}(\hat{\varphi}-\varphi) \stackrel{d}{\rightarrow} N\left(0, V_{\varphi}\right), \hat{V}_{\varphi} \stackrel{p}{\rightarrow} V_{\varphi}$ and the matrix $G_{\varphi}\left(\varphi, \vartheta_{0}\right)$ $V_{\varphi} G_{\varphi}\left(\varphi, \vartheta_{0}\right)^{\prime}$ is of full rank $k_{g}=k p .$. Then

$$
\begin{aligned}
\operatorname{MD}-\operatorname{AR}\left(\vartheta_{0}\right) & \stackrel{d}{\rightarrow} \chi^{2}\left(k_{g}\right) \\
\operatorname{MD}-\mathrm{K}\left(\vartheta_{0}\right) & \stackrel{d}{\rightarrow} \chi^{2}(2) \\
\operatorname{MD}-\mathrm{J}\left(\vartheta_{0}\right) & \stackrel{d}{\rightarrow} \chi^{2}\left(k_{g}-2\right)
\end{aligned}
$$

where " $\stackrel{d}{\rightarrow}$ " and " $\stackrel{p}{\rightarrow}$ " indicate convergence in distribution and in probability, respectively, and $\chi^{2}(\kappa)$ indicates a chi square distribution with $\kappa$ degrees of freedom.

The proof of the proposition is given in the Appendix. Note that the assumptions we use are strictly weaker than the assumptions used to establish that classical MD $t$ statistics are asymptotically normal, because the latter require in addition the identification condition that the Jacobian matrix $G_{\vartheta}(\varphi, \vartheta)$ should be of full rank, see Newey and McFadden (1994). The assumptions in Proposition 1 do rule out some interesting cases, such as unit roots in the VAR, but this limitation applies also to the non-robust methods, see Moon and Schorfheide (2002). The development of methods that are robust both to weak instruments as well as near unit roots in the VAR coefficients is a topic for future research.

Identification-robust $(1-\alpha)$ level confidence regions for the parameters $(\theta, \varrho)$ can be obtained by inverting the MD-AR and MD-KJ tests, i.e., by collecting all the values of $\left(\theta_{0}, \varrho_{0}\right)$ that are not rejected by the tests at the $\alpha$ level of significance. 


\section{Comparison with GMM}

For clarity, we discuss a simple special case of the NKPC with only one parameter:

$$
\pi_{t}=E_{t} \pi_{t+1}+\lambda x_{t}+\varepsilon_{t}
$$

The reduced-form model for $\left(\pi_{t}, x_{t}\right)$ is given by:

$$
\left(\begin{array}{c}
\pi_{t} \\
x_{t}
\end{array}\right)=\left(\begin{array}{l}
\varphi_{1} \\
\varphi_{2}
\end{array}\right) x_{t-1}+\left(\begin{array}{l}
\eta_{t} \\
v_{t}
\end{array}\right)
$$

In this setting, the only relevant instrument is $x_{t-1}$, so the model is just-identified.

The distance function (5) is given by

$$
g(\hat{\varphi}, \lambda)=\hat{\varphi}_{1}\left(1-\hat{\varphi}_{2}\right)-\lambda \hat{\varphi}_{2}
$$

where $\hat{\varphi}$ is the OLS estimator of $\varphi$. For GMM, we use the moment condition $E\left[f_{t}(\lambda)\right]=0$, where $f_{t}(\lambda)=\left(\pi_{t}-\pi_{t+1}-\lambda x_{t}\right) x_{t-1}$, with corresponding sample moments:

$$
f_{T}(\lambda)=\frac{1}{T} \sum_{t=1}^{T} f_{t}(\lambda) .
$$

The GMM-based Anderson Rubin statistic for testing the null hypothesis $H_{0}: \lambda=\lambda_{0}$, is:

$$
\operatorname{GMM}-\operatorname{AR}\left(\lambda_{0}\right)=T f_{T}\left(\lambda_{0}\right)^{\prime} \hat{V}_{f f}\left(\lambda_{0}\right)^{-1} f_{T}\left(\lambda_{0}\right)
$$

where $\hat{V}_{f f}\left(\lambda_{0}\right)$ is an estimator of the asymptotic variance of $\sqrt{T} f_{T}\left(\lambda_{0}\right)$. Because $f_{t}(\lambda)$ is serially correlated, $\hat{V}_{f f}\left(\lambda_{0}\right)$ must be a heteroskedasticity and autocorrelation consistent (HAC) estimator. This is different from MD, where it suffices to use only a heteroskedasticityconsistent estimator of the variance of the reduced-form parameters, in view of the as- 
sumption that the proposed reduced-form VAR model represents the dynamics of $\left(\pi_{t}, x_{t}\right)$ adequately. Though this difference between MD and GMM is of no consequence asymptotically, it could be important in finite samples, as HAC estimators are known to cause size distortions in finite samples, see, e.g., Sun, Phillips, and Jin (2008).

The main difference that we wish to emphasize is in the way in which the above two approaches make use of the identifying restriction $E_{t-1} \varepsilon_{t}=0$. Specifically, the difference arises due to the presence of the forward-looking term $E_{t-1} \pi_{t+1}$ : GMM proxies $E_{t-1} \pi_{t+1}$ by projecting $\pi_{t+1}$ directly on the instrument $x_{t-1}$, while MD uses the iterative expectation based on the reduced-form model (14), namely $E_{t-1} \pi_{t+1}=\varphi_{1} \varphi_{2} x_{t-1}$. This makes it clear that GMM is more general, since the direct projection of $\pi_{t+1}$ on the instruments will remain valid (albeit inefficient) when the reduced-form model is misspecified, while the MD iterative forecast will be biased when the reduced-form model is misspecified. The distinction is akin to the issue of direct versus iterative multistep forecasts that has been studied in the forecasting literature, see Marcellino, Stock, and Watson (2006). Iterative forecasts are more efficient, but direct forecasts are more robust to misspecification of the forecasting model.

From the above discussion, we expect that when the additional structure imposed by the MD approach is correct, the resulting estimates of $E_{t-1} \pi_{t+1}$ will be more accurate, and this will lead to more efficient inference on the structural parameter $\lambda$. To get a sense of the extent of efficiency gains one might expect, we report the power curves for the MD and GMM tests of the hypothesis $H_{0}: \lambda=\lambda_{0}$ in equation (13). The power curves are computed by simulation based on the reduced form model given by equation (14), where $\eta_{t}=\varepsilon_{t}+\left(\lambda+\varphi_{1}\right) v_{t}$, and $\varepsilon_{t}, v_{t}$ are drawn from a zero-mean bivariate Normal distribution with unit variances and correlation $\rho_{\varepsilon v}=.2$.

The strength of identification of $\lambda$ can be measured by the concentration parameter $\mu^{2}$, see Stock, Wright, and Yogo (2002) for details. The interpretation of $\mu^{2}$ is most easily given in terms of the so-called first-stage $F$ statistic that tests the rank condition for identification, $\varphi_{2} \neq 0$ in this case. Under the null hypothesis of no identification, $\varphi_{2}=0$, the expected value 
of the first-stage $F$ statistic is equal to one, while under the alternative it is greater than one and it is approximately equal to $E(F)-1$. In the present example, $\mu^{2}=T \varphi_{2}^{2} /\left(1-\varphi_{2}^{2}\right)$, so the strength of identification depends only on $\varphi_{2}$.

We use a sample size of 200 observations and we consider two different values for the concentration parameter: $\mu^{2}=4$ and $\mu^{2}=100$, corresponding to weak and strong identification, respectively. The empirical moments for GMM are $f_{t}(\lambda)=Z_{t}^{\prime} \varepsilon_{t}$, with $Z_{t}=\left[x_{t-1}, \pi_{t-1}\right]$ and $Z_{t}=\left[x_{t-1}, \pi_{t-1}, x_{t-2}, \pi_{t-2}\right]$. This corresponds to a $\operatorname{VAR}(1)$ and $\operatorname{VAR}(2)$ reduced-form models for MD. For the GMM test, we use the Newey and West (1987) estimator for the variance while the MD tests are based on the OLS estimator for the reduced-form VAR coefficients and the White (1980) estimator of their variance. The results are reported in Figure 1.

We see that in both cases of weak and strong instruments, the MD-AR and MD-KJ

tests are substantially more powerful than their GMM counterparts. When the degree of overidentification is one, the MD-AR test is almost as powerful as the MD-KJ test, as expected, and the difference increases as the degree of overidentifcation gets higher. Also, the MD tests based on a VAR(1) reduced-form are more powerful than those based on a $\operatorname{VAR}(2)$, since the former exploit the additional restriction that the second order lags are irrelevant, which is consistent with the DGP (14). So, the power of the tests is increasing in the number of correctly specified restrictions.

\section{Empirical results}

We use quarterly data on inflation and the labor share over the period 1984 to 2008. Inflation is calculated from the quarterly GDP deflator, while the labor share is obtained from the Bureau of Labor Statistics and transformed according to procedure used in Sbordone (2002) that was also used by Galí and Gertler (1999).

We focus on the post-1983 sample so as to avoid issues of instability in the dynamics of 
$\mu^{2}=4,2$ instruments
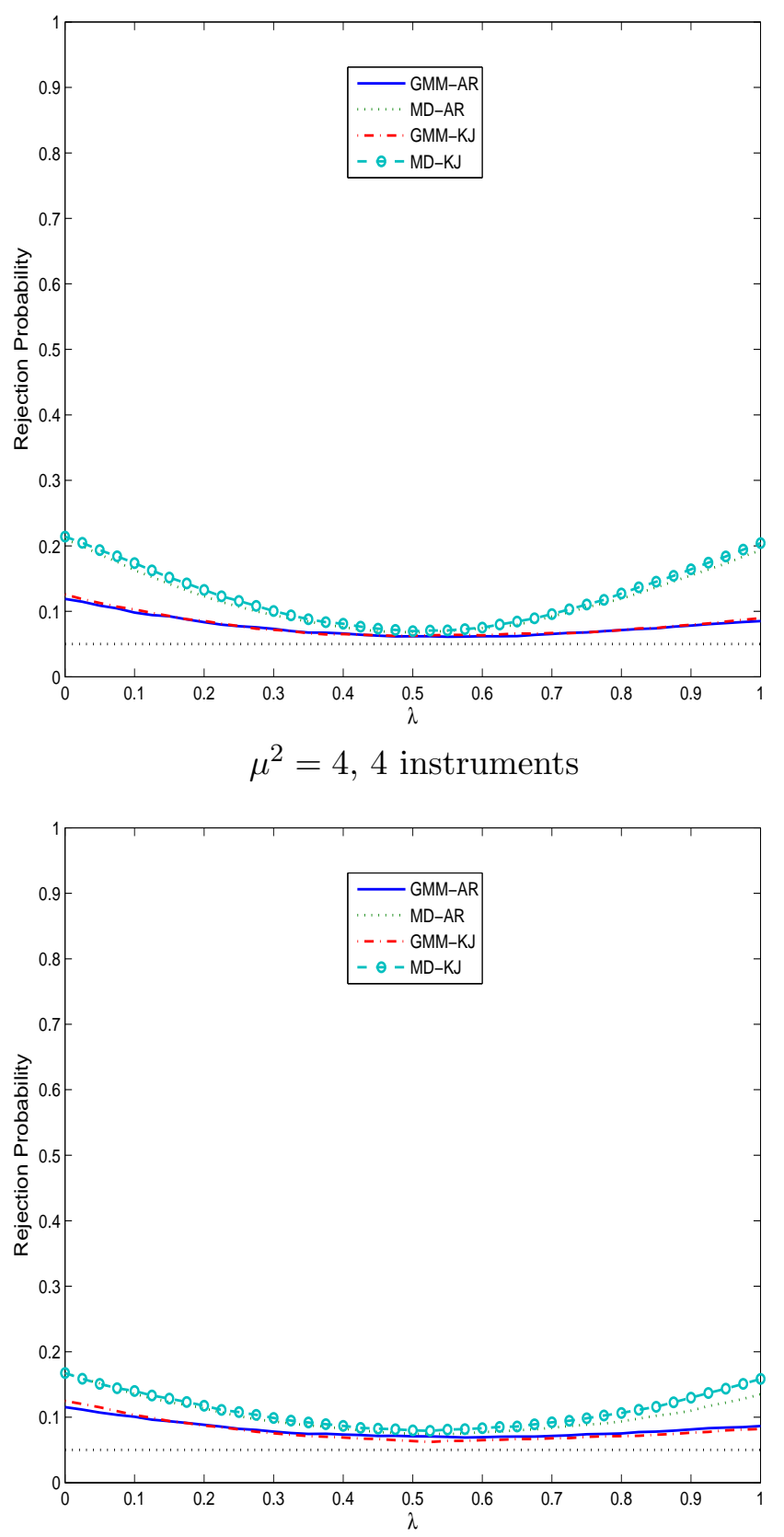

$\mu^{2}=100,2$ instruments

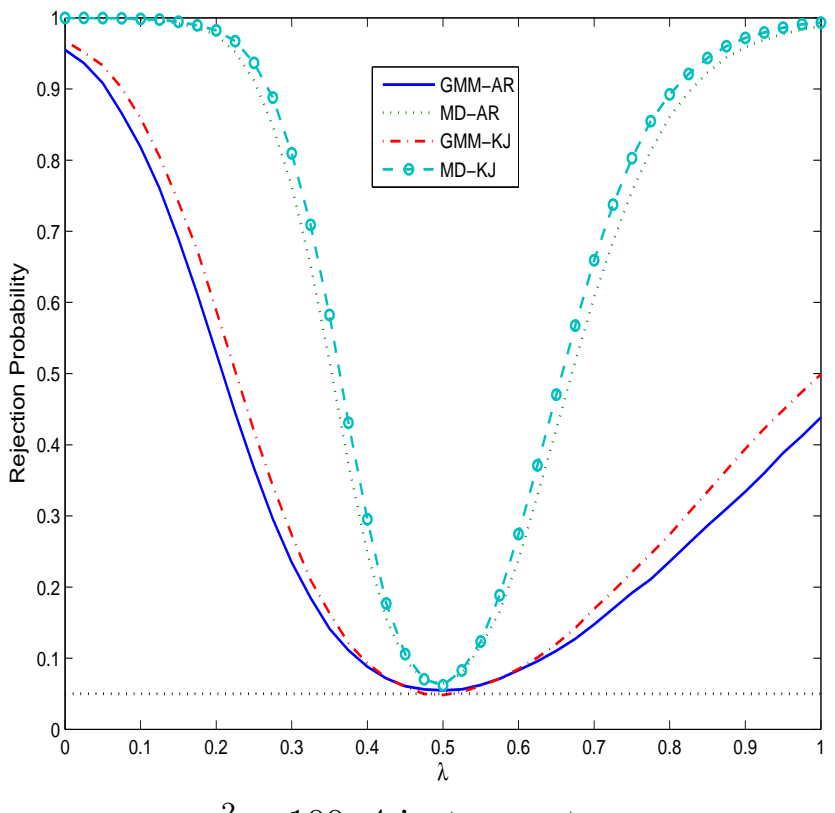

$\mu^{2}=100,4$ instruments

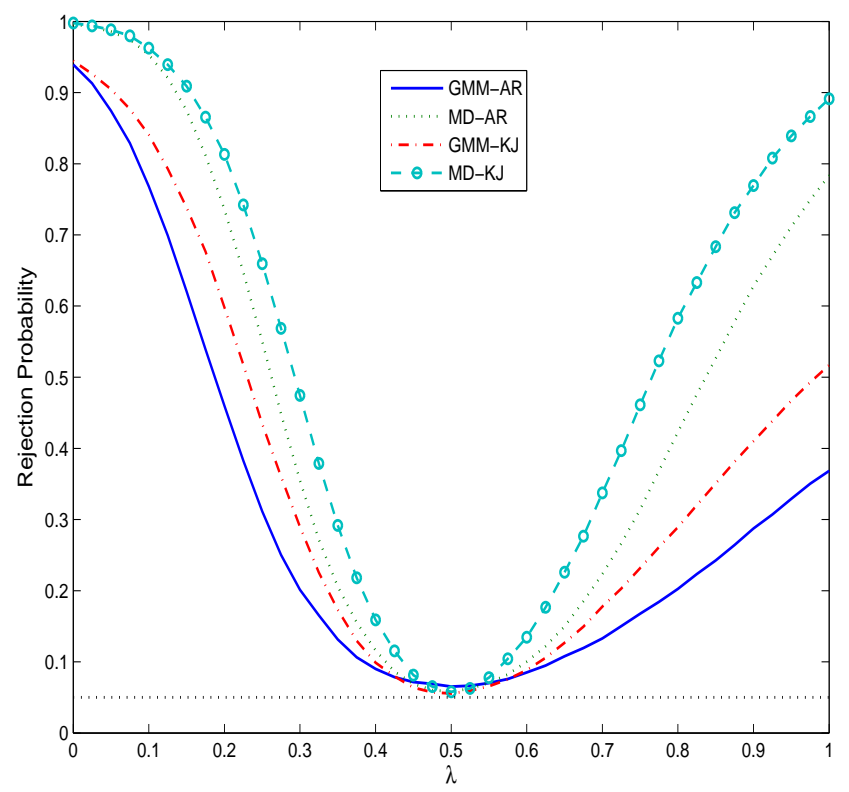

Figure 1: Rejection probability using 5\% significant level for testing $H_{0}: \lambda=0.5$ against $H_{1}: \lambda \neq 0.5$, and $\rho_{\varepsilon v}=0.2$. The sample size is 200 and the number of Monte Carlo replications is 10,000 . 
inflation and the labor share induced by, amongst other things, changes in monetary policy in the late 1970s and early 1980s. This is in line with the recent literature, see e.g., Krause, Lopez-Salido, and Lubik (2008) and is also further motivated by the stability tests reported in Kleibergen and Mavroeidis (2009), which indicate instabilities before 1984 .

Confidence sets for the price stickiness and indexation parameters $(\theta, \varrho)$ are computed by grid search within the parameter space $\theta, \varrho \in[0,1]$. Figures 2 and 3 report $95 \%$ and $90 \%$ level confidence sets based on inverting the AR tests and the KJ combination tests defined in section 2 and contrast them with their GMM counterparts. The MD tests are based on a VAR(3) for inflation and the labor share, and GMM accordingly uses three lags of both of these variables as instruments, following Sbordone (2005) and Kleibergen and Mavroeidis (2009). ${ }^{5}$ The efficient weight matrices for MD and GMM are based on the White (1980) and Newey and West (1987) estimators, respectively, as discussed in section 3.

MD-AR

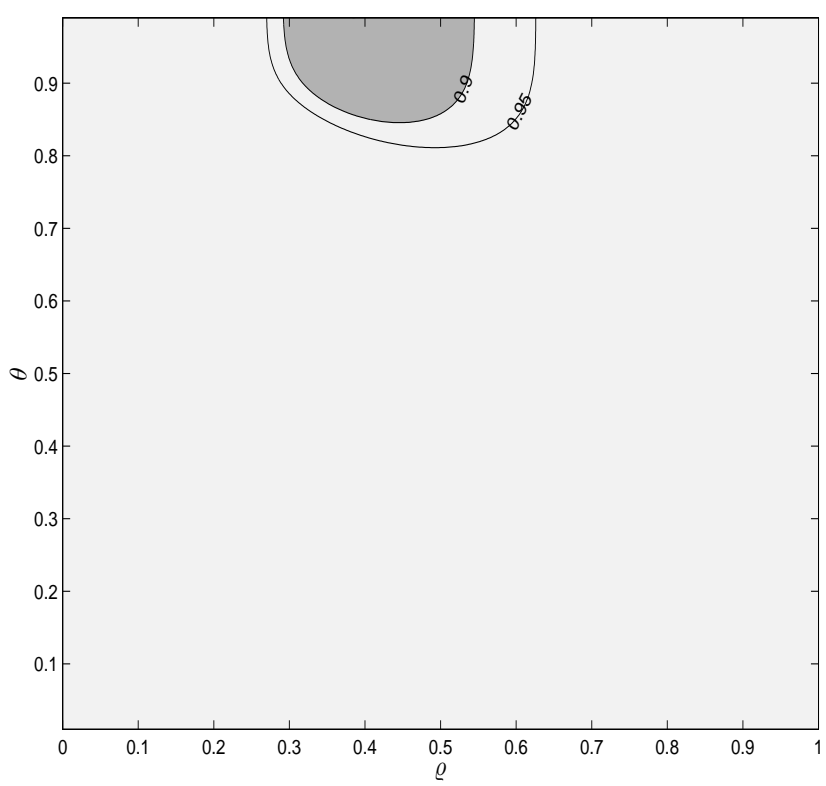

GMM-AR

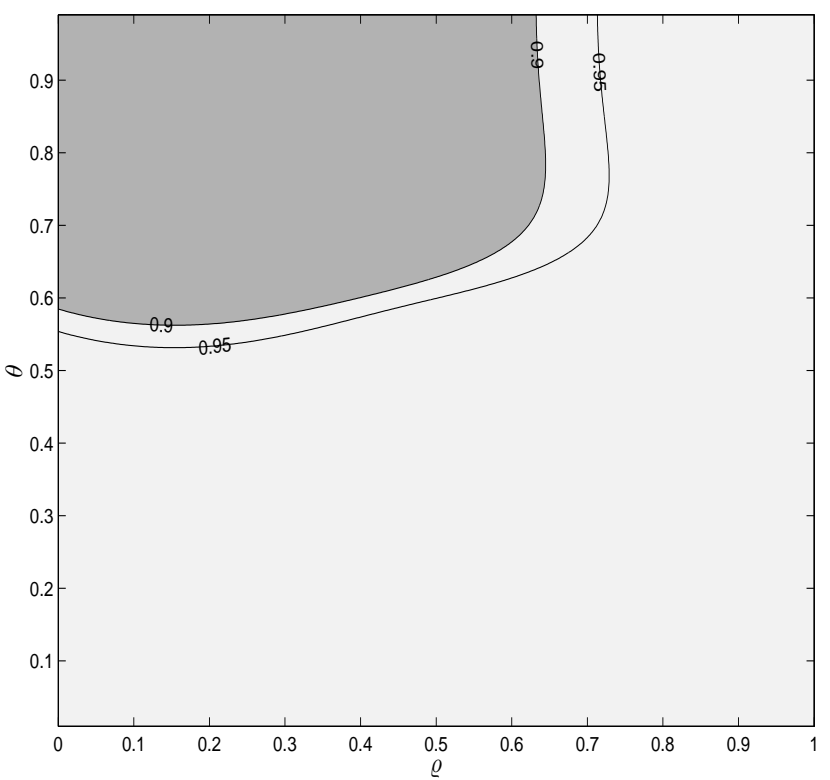

Figure 2: MD-AR and GMM-AR confidence sets for the parameters $(\theta, \varrho)$ of the NKPC. The dark and light gray areas are, respectively, the $90 \%$ and $95 \%$ confidence sets. The sample is $1984 \mathrm{q} 1-2008 \mathrm{q} 3$

The following conclusions emerge from these pictures. On the methodological side, we

\footnotetext{
${ }^{5}$ The results reported using the MD approach is not sensitive to the order of the VAR lag. 
MD-KJ

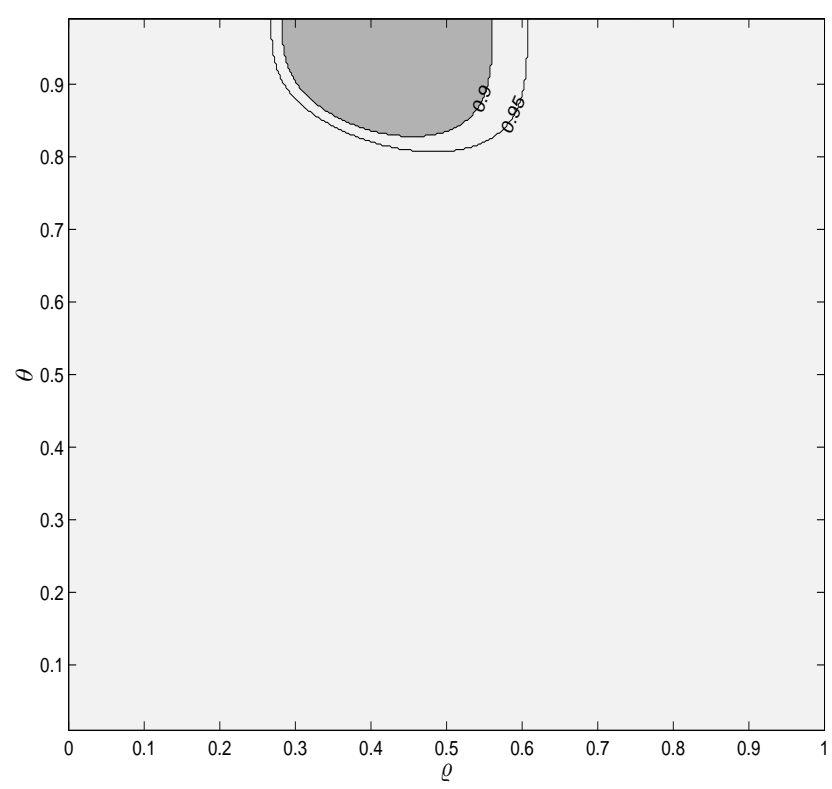

GMM-KJ

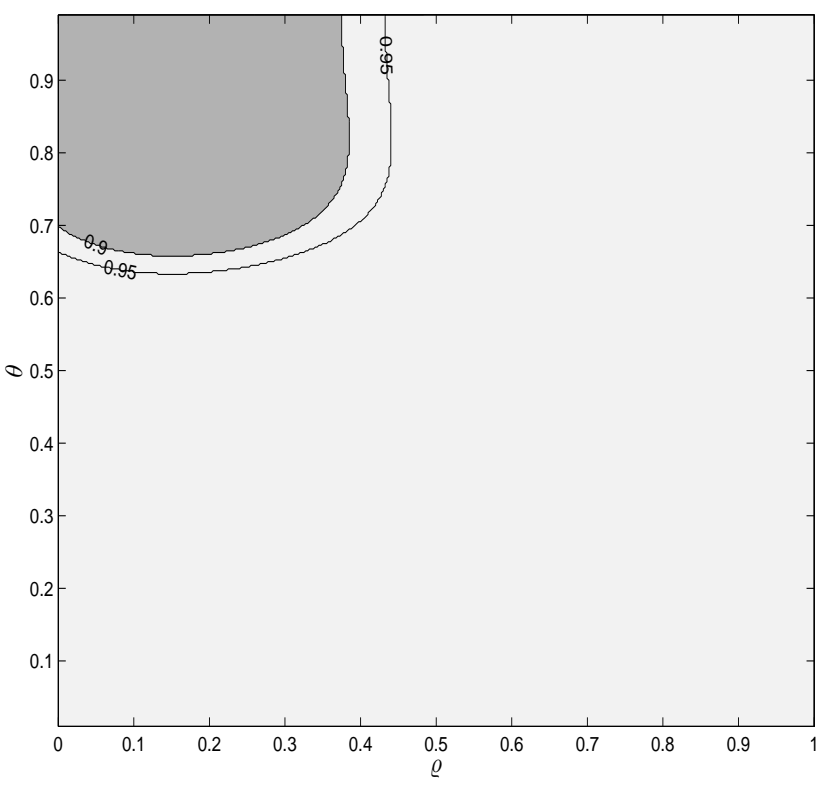

Figure 3: MD-KJ and GMM-KJ confidence sets for the parameters $(\theta, \varrho)$ of the NKPC. The dark and light gray areas are, respectively, the $90 \%$ and $95 \%$ confidence sets. The sample is 1984q1 - 2008q3

see that the confidence sets based on MD-AR test is less than half the size of their GMM counterpart, and a similar difference appears for the KJ sets. Thus, there are substantial power gains that arise from imposing the full-information restrictions, which are consistent with the simulations reported in section 3 .

On the empirical side, we can infer from both the MD-AR and MD-KJ 90\% sets that the indexation parameter lies roughly between 0.3 and 0.6. This indicates that indexation is significant, thus rejecting the pure forward-looking version of the NKPC, but that it is also less than 100\%, as in the model of Christiano, Eichenbaum, and Evans (2005). With regards to the parameter $\theta$ that measures the degree of price stickiness, we also find that it is more precisely estimated by MD than by limited-information GMM, with the confidence intervals spanning the region $4 / 5$ to 1 . However, in terms of the average duration of prices, i.e., the average time over which prices remain fixed, which is given by $1 /(1-\theta)$, we see that the confidence sets remain unbounded from above, as in the GMM case. One implication of the fact that $\theta=1$ is in the confidence set is that the slope of the NKPC is not significantly 
different from zero. This is also found in the limited-information studies cited above, and could be interpreted either as evidence that the labor share is not the relevant variable that drives inflation, or as evidence that the average duration of prices cannot be well-identified by aggregate macroeconomic data.

\section{Conclusion}

This paper was motivated by the findings in the literature that the new Keynesian Phillips curve is not well-identified. This finding was based on the use of limited-information methods, which make minimal assumptions about the dynamics of the labor share. We asked whether imposing the assumption that the dynamics of inflation and the labor share can be represented by a finite-order VAR model improves the identification of the NKPC parameters. We proposed two different methods for answering this question. These are based on the Minimum Distance approach used by Sbordone $(2002,2005)$ for the NKPC, but they do not require any assumption about identification, and therefore results derived from them are reliable even when identification fails. These methods are adaptations of the tests developed for GMM by Stock and Wright (2000) and Kleibergen (2005), which were recently developed in the context of limited dependent variable models by Magnusson (2008).

We found that the full-information approach improves significantly the identification of at least one of the two key structural parameters of the NKPC. Specifically, we find evidence of partial but not full indexation of prices to past inflation. Sharper inference relative to limited-information methods is obtained also for the parameter governing the average duration of prices, but the confidence intervals on this parameter remain unbounded from above, indicating that it remains weakly identified.

The results of this paper also suggest there are substantial efficiency gains in estimating forward-looking models by full- versus limited-information methods when the possibility of weak identification is taken into account. The methodology used here can be extended 
to estimate multiple-equation models, such as the dynamic stochastic general equilibrium (DSGE) model of Christiano, Eichenbaum, and Evans (2005). This is an important topic for future research, as DSGE models are currently estimated using procedures that are not robust to possible identification failure.

\section{A Appendix}

Proof of proposition 1 The asymptotic distribution of MD-AR $\left(\vartheta_{0}\right)$ follows by applying the Delta method. For the distribution of the MD-K and MD-J statistics, it suffices to show that the matrix $\hat{D}(\vartheta)$ defined in equation (10) is asymptotically independent of $g(\hat{\varphi}, \vartheta)$. First, note that the $k p \times k p$ matrix $A(\varphi)$ in the reduced-form VAR (3) can be written as $A(\varphi)=B \Phi+C$ where $B=\left(I_{k}, 0, \ldots, 0\right)^{\prime}$ is $k p \times k$ matrix, $\Phi=\left(\Phi_{1}, \ldots, \Phi_{p}\right)$ is $k \times k p$ matrix of the $\operatorname{VAR}(\mathrm{p})$ coefficients, and $C=\left(0,0 ; I_{k p-1}, 0\right)$ is $k p \times k p$ and $\varphi=\operatorname{vec}(\Phi)$. So $A(\varphi)$ is linear in $\varphi$, and the distance function (5) is differentiable and its Jacobian w.r.t. $\varphi$ is given by:

$$
G_{\varphi}(\varphi, \vartheta)=I_{k p} \otimes\left(e_{\pi}-\frac{(1-\theta)^{2}}{\theta(1+\varrho)} e_{x}-\frac{1}{1+\varrho} A(\varphi) e_{\pi}\right)^{\prime} B-\frac{1}{1+\varrho}\left[A(\varphi)^{\prime} \otimes e_{\pi}^{\prime} B\right] .
$$

Clearly, $\frac{\partial G_{\varphi}(\hat{\varphi}, \vartheta)}{\partial \theta}$ and $\frac{\partial G_{\varphi}(\hat{\varphi}, \vartheta)}{\partial \varrho}$ exist and are linear in $\hat{\varphi}$. Hence, by a first-order Taylor expansion around $\varphi$ we obtain: $\hat{\xi}_{g} \equiv \sqrt{T} g(\hat{\varphi}, \vartheta)=G_{\varphi}(\varphi, \vartheta) \xi_{\varphi}+o_{p}(1), \hat{\xi}_{\theta} \equiv \sqrt{T}\left[\frac{\partial g(\hat{\varphi}, \vartheta)}{\partial \theta}-\frac{\partial g(\varphi, \vartheta)}{\partial \theta}\right]$ $=\frac{\partial G_{\varphi}(\varphi, \vartheta)}{\partial \theta} \xi_{\varphi}+o_{p}(1)$ and $\hat{\xi}_{\varrho} \equiv \sqrt{T}\left[\frac{\partial g(\hat{\varphi}, \vartheta)}{\partial \varrho}-\frac{\partial g(\varphi, \vartheta)}{\partial \varrho}\right]=\frac{\partial G_{\varphi}(\varphi, \vartheta)}{\partial \varrho} \xi_{\varphi}+o_{p}(1)$, where $\xi_{\varphi} \sim$ $N\left(0, V_{\varphi}\right)$. Thus, $\left(\hat{\xi}_{g}: \hat{\xi}_{\theta}: \hat{\xi}_{\varrho}\right) \stackrel{d}{\rightarrow}\left(\xi_{g}: \xi_{\theta}: \xi_{\varrho}\right)$, where $\xi_{g}, \xi_{\theta}$ and $\xi_{\varrho}$ are $k p$-dimensional jointly normally distributed random vectors with covariances $E\left(\xi_{\theta} \xi_{g}^{\prime}\right) \equiv V_{\theta g}(\vartheta)=\frac{\partial G_{\varphi}(\varphi, \vartheta)}{\partial \theta} V_{\varphi} G_{\varphi}(\varphi, \vartheta)^{\prime}$ and $E\left(\xi_{\varrho} \xi_{g}^{\prime}\right) \equiv V_{\varrho g}(\vartheta)=\frac{\partial G_{\varphi}(\varphi, \vartheta)}{\partial \varrho} V_{\varphi} G_{\varphi}(\varphi, \vartheta)^{\prime} \cdot \hat{V}_{\theta g}(\vartheta)$ and $\hat{V}_{\varrho g}(\vartheta)$, defined below equation 10 , are consistent for $V_{\varrho g}(\vartheta)$ and $V_{\varrho g}(\vartheta)$, respectively, by Slutsky's theorem, since $V_{\varrho g}(\vartheta)$ and $V_{\varrho g}(\vartheta)$ are continuous in $\varphi$.

Let the $k p \times 2$ matrix $\hat{D}(\vartheta)$ defined in the text be written as $\hat{D}(\vartheta)=\left[\hat{D}_{\theta}(\vartheta), \hat{D}_{\varrho}(\vartheta)\right]$. 
Under strong identification, $\hat{D}(\vartheta) \stackrel{p}{\rightarrow} G_{\vartheta}(\varphi, \vartheta)$ has full rank. So, $\hat{D}(\vartheta)$ is trivially independent of $g(\hat{\varphi}, \vartheta)$. Under weak identification, we can use the nesting $G_{\vartheta}(\varphi, \vartheta)=T^{-1 / 2} D$, where $D=\left[D_{\theta} \vdots_{\varrho}\right]$ is a nonrandom matrix. This implies that $\sqrt{T} \hat{D}_{\theta}(\vartheta)=\xi_{\theta . g}+o_{p}(1)$, where $\xi_{\theta . g}=\xi_{\theta}-V_{\theta g}(\vartheta) V_{g g}(\vartheta)^{-1} \xi_{g}+D_{\theta}$, and $\xi_{\theta . g}$ is independent of $\xi_{g}$, and similarly for $\hat{D}_{\varrho}(\vartheta)$. Thus, $\sqrt{T} \hat{D}(\vartheta)$ is asymptotically independent of $\sqrt{T} g(\hat{\varphi}, \vartheta)$. Hence, in both cases, $\left[\hat{D}(\vartheta)^{\prime} \hat{V}_{g g}(\vartheta)^{-1} \hat{D}(\vartheta)\right]^{-1 / 2} \hat{D}(\vartheta)^{\prime} \hat{V}_{g g}(\vartheta)^{-1} \sqrt{T} g(\hat{\varphi}, \vartheta)$ is approximately normal with identity variance matrix. The asymptotic distribution of MD-K and MD-J follows by the continuous mapping theorem.

\section{References}

Anderson, T. W. and H. Rubin (1949). Estimation of the parameters of a single equation in a complete system of stochastic equations. Ann. Math. Statistics 20, 46-63.

Calvo, G. A. (1983). Staggered prices in a utility maximizing framework. Journal of Monetary Economics 12, 383-398.

Christiano, L. J., M. Eichenbaum, and C. Evans (2005). Nominal rigidities and the dynamic effects of a shock to monetary policy. J. Political Economy 113, 1-45.

Dufour, J.-M., L. Khalaf, and M. Kichian (2006). Inflation dynamics and the new Keynesian Phillips curve: An identification robust econometric analysis. Journal of Economic Dynamics and Control 30(9-10), 1707-1727.

Dufour, J.-M., L. Khalaf, and M. Kichian (2007). Structural multi-equation macroeconomic models: A system-based estimation and evaluation approach. Discussion paper, Bank of Canada.

Dufour, J.-M., L. Khalaf, and M. Kichian (2008). On the precision of the Calvo parameter in structural NKPC models. Technical report.

Fuhrer, J. C. and G. R. Moore (1995). Inflation persistence. Quarterly Journal of Eco- 
nomics 110, 127-159.

Galí, J. and M. Gertler (1999). Inflation dynamics: a structural econometric analysis. Journal of Monetary Economics 44, 195-222.

Hansen, L. P., J. Heaton, and A. Yaron (1996). Finite sample properties of some alternative GMM estimators. Journal of Business and Economic Statistics 14, 262-280.

Kleibergen, F. (2002). Pivotal statistics for testing structural parameters in instrumental variables regression. Econometrica $70(5), 1781-1803$.

Kleibergen, F. (2005). Testing parameters in GMM without assuming that they are identified. Econometrica 73(4), 1103-1123.

Kleibergen, F. and S. Mavroeidis (2009). Weak Instrument Robust Tests in GMM and the New Keynesian Phillips Curve. Journal of Business and Economic Statistics. forthcoming.

Krause, M. U., D. Lopez-Salido, and T. A. Lubik (2008). Inflation dynamics with search frictions: A structural econometric analysis. Journal of Monetary Economics 55(5), $892-916$.

Magnusson, L. M. (2008). Inference in limited dependent variable models robust to weak identification. Working Papers 0801, Tulane University, Department of Economics.

Marcellino, M., J. H. Stock, and M. W. Watson (2006). A comparison of direct and iterated multistep ar methods for forecasting macroeconomic time series. Journal of Econometrics 135(1-2), 499-526.

Newey, W. K. and D. McFadden (1994). Large sample estimation and hypothesis testing. In R. F. Engle and D. McFadden (Eds.), The Handbook of Econometrics, Volume 4, pp. 2111-2245. North-Holland.

Moon, H., and F. Schorfheide (2002). Minimum Distance Estimator of Nonstationary Time Series Models. Econometric Theory 18(6), 1385-1407. 
Newey, W. K. and K. D. West (1987). A simple, positive semidefinite, heteroskedasticity and autocorrelation consistent covariance matrix. Econometrica 55(3), 703-708.

Sbordone, A. M. (2002). Prices and unit labor costs: a new test of price stickiness. Journal of Monetary Economics 49, 265-292.

Sbordone, A. M. (2005). Do expected future marginal costs drive inflation dynamics? Journal of Monetary Economics 52(6), 1183-1197.

Schorfheide, F. (2005). VAR forecasting under misspecification. Journal of Econometrics 128(1), 99-136.

Stock, J. H. and J. H. Wright (2000). GMM with weak identification. Econometrica 68(5), $1055-1096$.

Stock, J. H., J. H. Wright, and M. Yogo (2002). GMM, weak instruments, and weak identification. Journal of Business and Economic Statistics 20, 518-530.

Sun, Y., P. C. B. Phillips, and S. Jin (2008). Optimal bandwidth selection in heteroskedasticity-autocorrelation robust testing. Econometrica 76(1), 175-194.

White, H. (1980). A heteroskedasticity-consistent covariance matrix estimator and a direct test for heteroskedasticity. Econometrica 48(4), 817-38.

Woodford, M. (2003). Interest and Prices: Foundations of a Theory of Monetary Policy. Princeton University Press. 